\title{
Uebersicht der vier Typen von regenscheuen Blüten, deren Pollenschutz etc. auf einem phytodynamischen Principe beruht. ')
}

\section{Von Prof. Dr. A. Hansgirg in Prag.}

I. Typus. Pflanzen, deren Blüten bei Regenwetter ihre Perianthien so schliessen, dass ein Eindringen der Regentropfen in die Blüten erschwert wird oder nicht stattfinden kann, wobei die auf steifen, nicht regenscheu krümmungsfähigen Stielen sitzenden Blüten oder Blütenköpfchen ihre Lage nicht verändern.

Hierher gehören von Monocotylen einige Liliaceen (z. B. Tulipa, Ornithogalum, Erythronium u. ä.), Irideen (Crocus, Romulea u. ä.), Amaryllideen (Sternbergia), Colchicaceen (Colchicum), Gramineen und Juncaceen.

Von Dicotylen seien hier folgende Familien beispielsweise angeführt: Compositen (Catananche, Sphenogyne, Venidium, Hymenostoma, Crepis, Hieracium, Carlina und zahlreiche andere Compositen, deren Blütenköpfchen sich periodisch öffnen und schliessen), Campanulaceen (Specularia, Campanula), Gentianaceen (Gentiana, Erythraea, Chironia), Polemoniaceen (Gilia, Leptosiphon, Collomia), Solanaceen (Mandragora, Datura), Ficoideen (Mesembryanthemum), Ranunculaceen (Paeonia, Eranthis, Trollits, Anemone blanda, Ranunculus carpathicus u. ä.), Magnoliaceen (Magnolia), Nymphaeaceen (Nymphaea), Cactaceen (Opuntia, Mammillaria), Cruciferen (einige Draba-, Arabis-, Aubrietia-, Malcolmia-Arten u. ä.), Papaveraceen (Escholtzia, Sanguinaria), Portulacaceen (Portulaca), Rosaceen (Rosa, einige Potentilla-Arten), Malvaceen, Leguminosen, Oxalideen, Linaceen, Polygalaceen, Onagrarien u. ä., von welcheu jedoch viele, mit regenscheuen Blüten versehene Arten zum vierten Typus gehören.

II. Typus. Pflanzen, deren in der Anthese befindliche, auf biegsamen, meist vertical aufrechten und geraden Stielen sitzende Blüten mit ihrer Apertur zenithwärts gerichtet sind und bei eintretendem Regenwetter, obne ihr Perianthium zu schliessen, durch besondere regenscheue $\mathrm{Kr} u ̈ \mathrm{mmungen} \mathrm{der} \mathrm{die} \mathrm{einzelnen} \mathrm{Blüten}$ tragenden Blütenstiele ihren Pollen, Nectar etc. vor Benetzung durch Regen schützen und der Gefahr der Füllung ihrer Cor̀olle mit Wasser zu entgehen suchen.

Zu diesem Typus gehören blos solche Pflanzen, deren Blütenstiele, bezichungsweise -Stengel durch fallende Regentropfen, Veränderungen im Feuchtigkeitszustande der Luft, Anprall des Windes etc.

1) Der Verfasser wird in einer grösseren Arbeit, welche er zum Drucke vorbereitet, ansfährlich über die Blätenombrophobie, die Widerstandsfähigkeit des Pollens gegen Wasser, die gamo- und karpotropischen Bewegungen und diejenigen Krümmungen, mit welchen er sich bereits in seinen "Phytodynamischen Untersuchungen ", 1893 , beschäftigt hat $u$. s. w. abhandeln. 
jedoch nicht blos passiv infolge der Belastung mit Regentropfen, Thau etc. besoudere Krümmungen ausführen, z. B. einige Ranunculaceen (Ranunculus, Anemone), Rosaceen (Geum, Rubus, Fragaria), Geraniaceen, Papaveraceen, Linaceen, Caryophyllaceen (Dianthus u. ä.), Cruciferen, Leguminosen (Coronilla), Saxifragaceen (Saxifraga), Violaceen, Boragineen (Cynoglossum, Omphalodes), Convolvulaceen, Campanulaceen, Polemoniaceen, Solanaceen u. ä.

III. Ty pus. Pflanzen, deren Blütenstände sich durch besondere Krümmungen der Blütenstandachse oder der als Träger der Blütenköpfchen oder Dolden etc. dienenden Achsen, insbesondere der soeben in der Anthese befindlichen Blüten tragenden Endtheile der Blütenspindel vor dem Regen zu schützen suchen.

Hierher gehören zahlreiche Cruciferen (einige Alyssum-, Drabu-, Arabis-, Kernera-, Eunomia-, Erysimum-, Iutchinsia-, Rapistrum-, Crambe-Arten u. ä.). Von Fumariaceen, z. B. Corydalis rosea. Von Compositen einige Cenia-, Emilia-, Leptosyne-, Coreopsis-, Quizotia-, Lasthenia-, Ptilomeris-, Laya-, Galinsogaea-Arten u. ä. Von Dipsaceen einige Scabiosa-, Knautia-, Cephalaria- und PterocephalusArten. Von Umbelliferen und Euphorbiaceen die meisten, wenn nieht alle Arten mit periodische Bewegungen ausführenden Doldenträgern.

IV. Ty pus. Pflanzen, deren bei schönem Wetter aufrecht gestelite und geöffnete Blüten bei eintretendem Regenwetter ihre Perianthien nicht blos schliessen, sondern gleichzeitig auch durch besondere erdwärts gerichtete Krümmungen der Blütensti el e oder der stie]artigen Fruchtknoten (Köpfchenstiele etc.) schützen und von der Bichtung der einfallenden Regentropfen wegkrümmen.

Hierher gehören von Monocotylen blos einige Tulipa- und Brodiaea- (Triteleja-) Arten.

Von Dicotylen zahlreiche Campanulaceen, Hydrophyllaceen, (Nemophila), Polemoniaceen (Polemonium), Solanaceen (Solanum), Scrophulariaceen (Veronica), Convolvulaceen (Nolana, Convolvulus), Compositen (Bellis, Rhodanthe, Sonchus u. ä.), Primulaceen (Anagallis), viele Caryophyllaceen, Oxalideen, Linaceen, Cistineen (Helianthemum), Geraniaceen, Onagraceen (Epilobium, Kneiffia), Malvaceen (Palava, Malva), Rosaceen (Potentilla), Papaveraceen (Hypecoum), Limnantheen (Limnanthes), Ranunculaceen (Isopyrum, einige Anemone-, Hepatica-, Adonis-, Ranunculus-Arten), Cruciferen (Heliophila, Vesicaria, Cardamine, Biscutella, Thlaspi, Bunias u. a. $)^{1}$ )

') Einen fünften Typus könnten solche Pflanzen bilden bei welchen bei Regenwetter nicht blos die Blumenblätter über den Antheren sich zasammenschliessen, sondern auch die bei trockenem Wetter offenen Antheren sich schliessen, wie z. B. bei Bulbocodium u. ä. 